\title{
Seismic signature of the Contagem River (Brasilia), Brazil in dry and flooding days and its impacts on HVSR measurements
}

\section{Yawar Hussain, Susanne Maciel and Hans Balder Havenith}

\begin{abstract}
Rainfall-induced landslides may pose a significant risk to communities and infrastructures; the site characterization and natural period estimation therefore have a paramount importance in any risk assessment studies. The single station horizontal-to-vertical-spectral-ratio (HVSR) is a commonly adopted technique used to achieve these objectives. However, it is substantially impacted by the multiplicity of factors which may mislead the results, and hence their identification is crucial. In the present study, effects of fluvial systems on HVSR curves in dry and flooding days are studied based on the preliminary results obtained from a Brazilian case study. Data were acquired with a single short-period $(2 \mathrm{~Hz})$ seismometer in dry and rainy days installed within a seasonal stream in Ribeirão Contagem watershed of the Federal District of Brazil. The pre-processing of ambient noise records includes conversion from REFTEK to miniseed format and saving data in units of velocity after removing the instrumental response. First we analyzed pluviometric and seismic displacement data using change-point analysis to detect pattern changes. Then, the frequency content (spectrograms, percentiles), polarization attributes and changes in the HVSR peak and its amplitude as a result of variations in the ambient noise wave-fields induced by the river flow are analyzed. Additionally, the endmember modelling is also used for the quantification of the impacts of different climatological factors on HVSR measurements. At a particular frequency range the mean probabilistic power spectral density (PPSD) values changed during rainy days. The polarization analysis shows that most of the recorded energy arrived from the riverside. It is concluded that HVSR curves have their relation with the river-generated ambient noise and should be considered for any HVSR related studies. Future detailed studies are recommended for the detailed quantification of these seasonal river dynamics on the HVSR.
\end{abstract}

Keywords: End-member modelling; spectrograms; change-point analysis 
